2008

\title{
Advancing Epidemiological Science Through Computational Modeling: A Review with Novel Examples
}

Scott M. Duke-Sylvester

Eli N. Perencevich

Jon P. Furuno

Leslie A. Real

Holly Gaff

Old Dominion University, hgaff@odu.edu

Follow this and additional works at: https://digitalcommons.odu.edu/biology_fac_pubs

8 Part of the Computational Biology Commons, Environmental Sciences Commons, Veterinary Preventive Medicine, Epidemiology, and Public Health Commons, and the Zoology Commons

\section{Repository Citation}

Duke-Sylvester, Scott M.; Perencevich, Eli N.; Furuno, Jon P.; Real, Leslie A.; and Gaff, Holly, "Advancing Epidemiological Science Through Computational Modeling: A Review with Novel Examples" (2008). Biological Sciences Faculty Publications. 201.

https://digitalcommons.odu.edu/biology_fac_pubs/201

\section{Original Publication Citation}

Duke-Sylvester, S. M., Perencevich, E. N., Furuno, J. P., Real, L. A., \& Gaff, H. (2008). Advancing epidemiological science through computational modeling: A review with novel examples. Annales Zoologici Fennici, 45(5), 385-401. doi:10.5735/086.045.0503

This Article is brought to you for free and open access by the Biological Sciences at ODU Digital Commons. It has been accepted for inclusion in Biological Sciences Faculty Publications by an authorized administrator of ODU Digital Commons. For more information, please contact digitalcommons@odu.edu. 


\title{
Advancing epidemiological science through computational modeling: a review with novel examples
}

\author{
Scott M. Duke-Sylvester ${ }^{1}$, Eli N. Perencevich ${ }^{2,3}$, Jon P. Furuno ${ }^{2}$, \\ Leslie A. Real ${ }^{1}$ \& Holly Gaff ${ }^{4}$
}

1) Department of Biology, Emory University, Atlanta, GA 30322, USA

2) Department of Epidemiology and Preventive Medicine, University of Maryland School of Medicine, Baltimore, MD 21201, USA

3) Veterans Affairs Maryland Health Care System, Baltimore, MD 21201, USA

4) Department of Community and Environmental Health, Old Dominion University, Norfolk, VA 23529, USA

Received 7 Aug. 2008, revised version received 9 June 2008, accepted 9 June 2008

Duke-Sylvester, S. M., Perencevich, E. N., Furuno, J. P., Real, L. A. \& Gaff, H. 2008: Advancing epidemiological science through computational modeling: a review with novel examples. - Ann. Zool. Fennici 45: 385-401.

Computational models have been successfully applied to a wide variety of research areas including infectious disease epidemiology. Especially for questions that are difficult to examine in other ways, computational models have been used to extend the range of epidemiological issues that can be addressed, advance theoretical understanding of disease processes and help identify specific intervention strategies. We explore each of these contributions to epidemiology research through discussion and examples. We also describe in detail models for raccoon rabies and methicillin-resistant Staphylococcus aureus, drawn from our own research, to further illustrate the role of computation in epidemiological modeling.

\section{Introduction}

Epidemiology is defined as the study of the distribution, determinants and control of diseases and has been a cornerstone of public health research since the 19th century (Szklo \& Nieto 2000). Mathematical models of infectious diseases have played a significant part in that history beginning with Daniel Bernoulli's model for smallpox in 1760 (Blower \& Bernoulli 2004) and Ross's for malaria in 1897 (Ross 1911). Mathematical models are especially valuable for evaluating the consequences of experiments and interventions that would be unethical or cost-prohibitive to attempt under real world conditions.
Bernoulli, Ross and modelers that followed their initial contributions were, however, limited by the mathematics and computational approaches that could be undertaken by hand. Consequently, they were constrained in the range and type of questions they could ask.

The modern development of increased computational capacity, fortunately, has allowed us to consider models that would be impractical or impossible to analyze under the early constraint of analytic tractability. The emphasis in this paper is on the contributions made to infectious disease epidemiology through the incorporation of computational approaches to modeling the infectious disease process. The range of benefits 
extends from the merely methodological, e.g. it is considerably more efficient to determine the eigenvalues and eigenvectors for large matrices computationally, to the incorporation of very minute details into the transmission dynamics across thousands of individuals exposed during an epidemic, e.g. through the construction of individually based simulations (Grimm \& Railsback 2005).

To organize our discussion we have identified three broad categories where computational modeling has contributed to epidemiological science: (1) extending the range of epidemiological issues that can be addressed through modeling, (2) advancing theoretical understanding of disease processes, and (3) helping to identify and evaluate specific intervention strategies. In each case, we use particular models as examples to demonstrate the role and importance of computation in epidemiology.

Including additional biological detail is an important way in which a computational approach can extend the range of models. The simplifying assumptions used to make a model analytically tractable are sometimes extreme or unreasonable (Anderson \& May 1991). Typical examples include assuming that stochastic effects are small or that a population is spatially well mixed. Simplifying assumptions can also introduce difficulties in the applicability of model results to particular diseases or populations. The introduction of model simplifications is largely a reflection of limits on the numbers of variable, equations, computations and data that humans can effectively handle. These limitations are, of course, exactly the problems that computers are designed to address. By admitting more variables and equations, restrictive assumptions can be relaxed, more biological detail can be included and a model can more closely reflect reality. For example, a computer model can explicitly trace interactions between individuals based on proximity rather than assuming a population is spatially well mixed. Computer models also allow us to include additional components to an existing model, such as the effects of multiple interacting diseases (e.g. human immunodeficiency virus (HIV) and tuberculosis). Differences between basic and extended models have been used to demonstrate the relative importance of particular mechanisms such as age-structure (DeAngelis \& Waterhouse 1987).

A computational approach can also extend the range of models to include the effects of specific intervention strategies to control infectious disease. Since the prevention and control of disease is a central occupation of epidemiologists we consider it as a separate topic in our discussion of computational modeling. The tools of medicine and public health, such as antimicrobials, vaccines, and quarantine measures, have been enormously successful in treating, preventing, and controlling infectious disease. However, the use of these tools can lead to unexpected and undesirable outcomes such as the evolution of pathogens resistant to antimicrobials (O'Brien 2002). Computational models can include the effects of different control measures and allow us to evaluate alternative strategic uses of these health care measures (Anderson \& May 1990, Ferguson et al. 2001b, Smith et al. 2005b). Computational models allow us to evaluate in detail the way control measures and epidemiological processes interact to produce population outcomes. Another important application of modeling is as a tool to systematically address difficulties inherent in observing the effects of health care measures. The most appropriate assessment of the effectiveness of a health care strategy is not always clear a priori. Computational models can be used to evaluate rapidly different measures of success and provide a relative assessment of which are most likely to be useful. In this way models provide a repeatable, mathematically rigorous and quantitatively precise evaluation of public health policies. For example, Anderson and May (Anderson \& May 1991) used a computational model to evaluate the effects of observed patterns of age-dependent transmission on vaccination policy.

A model can be as simple and straightforward as a linear regression, or as complex as a spatially-explicit, individual-based simulation (Grimm \& Railsback 2005). Geographic Information Systems (GIS) models are used to combine numerous spatial data layers to model the potential spatial distribution of an infectious disease (Clarke et al. 1996). Sophisticated approaches to reconstructing the phylogeny of infectious agents and their hosts have been cen- 
tral in understanding disease processes (Biek et al. 2007). Individual based models take into account individual variability and behaviors (DeAngelis \& Mooij 2005, Grimm \& Railsback 2005).

In our examples, we have chosen a broad definition of what constitutes a computational model. We have included models that are considered mathematical, such as systems of differential equations, as well as models that can only be meaningfully classified as computational, such as individual-based models. It is difficult to draw a clean distinction between these categories. A model expressed as equations could be classified as computational if its results and conclusions are based solely on computer output. In gathering this range of models we have focused on examples in which a computer is central to revealing the relationship between determinants of infectious disease and the resulting distribution.

Authors often refer to their models as mathematical, even when most or all of the model analysis is based on computational results. Labeling such models as mathematical arises because their formulation follows directly from the long tradition of mathematical models that preceded modern personal computers. Examples include models based on systems of differential equations, finite difference equations or Leslie matrix models. However, many of the models that are derived from the mathematical tradition involve adding terms or equations that make it difficult or impossible to derive meaning from the model using traditional analytical techniques such as equilibrium analysis. In those cases, the dynamics are revealed numerically and conclusions are based on the analysis of computer output. On this basis we have included mathematical models that highlight the role of computational modeling in epidemiology.

In contrast to the mathematical models, approaches like individual-based modeling only became viable following the advent of modern computers. These models allow for individual variation within the population and track the fate of each member. These models require the rapid storage and retrieval of vast amounts of data, and extensive calculations to update each individual from one time step to the next. Obtaining results from this class of model requires the speed, accuracy and memory provided by computers. This class of models is clearly computational, and there is rarely any ambiguity about their status as such. As with the mathematical models we have included, the results and conclusions from these models rest upon simulation output.

To illustrate the points outlined above, we discuss two specific computational models drawn from our own research. In the firts example, we describe an individual-based patient model within a small ward of a hospital. This model is used to evaluate how individual patient variation interacts with different hospital policies designed to manage nosocomial, i.e., hospital-acquired, infections. The second example describes the application of a spatial optimal control model to the vaccination of wildlife to limit the epidemic spread of an infectious disease, namely rabies. In each case, we highlight the advantages of taking a computational approach.

\section{Contributions of computational models}

\section{Computational models extend the range of models that can be considered}

The addition of population structure is an important way in which a computational approach extends the range of models that can be considered. Adding population structure makes the model more closely reflect the biology, ecology or epidemiology of a particular system. A better correspondence between model and biology is important when variation within the population, such as differences in death rates between age groups, is an important mechanism controlling population dynamics. Additional structure and realism in a model can change our estimate of system dynamic and, in particular, how epidemiological dynamics will respond to human intervention to control disease spread as well as threats such as global warming and habitat destruction (Weiss \& McMichael 2004, Patz et al. 2004).

The disease status of individuals is an essential form of population structure in epidemiological modeling. Many epidemiology models start 
by stratifying the population by disease status with the standard classification of susceptible (S), infected (I) and recovered (R). That is, the population is thought of as being divided into three distinct groups. The susceptible group is generally defined to be those individuals that have never been infected but may become infected later. The infected group is those individuals that are infected and are capable of infecting others. Finally, the recovered group is composed of those individuals that have cleared the pathogen. Models that structure populations in this way are referred to as compartment models (Anderson \& May 1991) and often receive specific designations that reflect specific structures. A model with susceptible, infected and recovered individuals is an SIR-model. In these models, the disease status delineates groups with substantially different demographic parameters that are important determinants of disease spread. For example, members of these groups may have different fecundities, dispersal patterns, life expectancies, risk of reinfection and may differ in the rate of mortality.

However, many diseases cannot be modeled by a simple SIR structure but rather require multiple infectious classes. The number of divisions used can reflect many factors, including different clinical manifestations, various levels of immunity after recovery or whether an infection is treatable with antibiotics or is refractory. Kim et al. (2007) explicitly modeled multiple strains of human papillomavirus (HPV) to evaluate alternative screening strategies since only certain strains of HPV are cancer-causing. Stilianakis et al. (1998) developed a model for influenza with two susceptible classes (with and without chemoprophylaxis) and nine infectious classes that include treated and untreated individuals, symptomatic and asymptomatic individuals, and patients infected with either a wild type or a resistant virus. Stilianakis et al. (1998) used their model to show that, relative to treating symptomatic individuals, chemoprophylaxis was more successful at reducing the number of influenza infections, the duration of an epidemic and limiting the development of drug resistance.

Additional forms of population structure, such as spatial, age or class structure can also be important features to incorporate into models.
Ball and Lyne (2002) showed that dividing a population into individual households is valuable when determining optimal vaccination strategies. Hyman and LaForce (2003) added the spatial structure of a 33-city network to an influenza model to investigate the spread of epidemics within and among each city. Boender et al. (2007) used a computational model that included the location of 5360 poultry farms to analyze the 2003 outbreak of avian influenza in the Netherlands. Using this model, they showed that the spatial distribution of infected farms could be accounted for by variation in farm density (Boender et al. 2007).

Seasonality is among the most important environmental processes driving disease dynamics (Altizer et al. 2006). Periodic cycles in case numbers have been observed for many infectious diseases, including influenza (Cox \& Fukuda 1998) and rotavirus infections (Cook et al. 1990). Seasonal variation in the number of reported cases has been attributed to several causes, including seasonal temperature changes, changes in rainfall and seasonal cycles in the rate of contact between hosts (Dowell 2001). Computational modeling allows for the inclusion of seasonality in the environment and evaluation of alternative hypothesized links between seasonality and cycles in host/pathogen dynamics. Computational modeling has been used to make a strong case that variation in annual cycles of measles cases among school-aged children is driven by seasonal aggregation of students during the school year (Grenfell 1992, Bolker 1993, Earn et al. 2000). Recently, Ferrari et al. (2008) used a model to show that cycles in the number of measles cases in Africa can be produced by season variation in rainfall.

Demographic stochasticity can be an important element determining population dynamics, especially when numbers are relatively small. This is important from an epidemiological perspective when the prevalence of a disease is low and the susceptible population is small. Whether or not a disease persists under these conditions depends, in part, on the occurrence of rare transmission events. A number of models have been developed to investigate the role of demographic and environmental stochasticity on disease dynamics; however, even relatively simple sto- 
chastic models are difficult to analyze analytically and a computational approach is often required (Anderson \& May 1991, Renshaw 1991).

The type of additional structure needed for a given model will depend on both the specific disease and the questions being asked. For example, a model of influenza can divide a population into distinct age classes for school-aged children and adults to include age-specific patterns of interaction that may account for age specific patterns in the likelihood of infection. Similarly, we can add spatial heterogeneity that reflects real world patterns. Another example would be to allow specific parameter values to vary according to a seasonally varying function (Bacaer 2007). With the advances in satellite imagery and other GIS datasets, parameter values can be tied directly to measured data points. For example, mosquito development time can be defined as a function of average daily temperature for each one-degree grid cell across a defined landscape. In addition to population structure, a model can incorporate interactions with predators or prey. It may also be important to include the effects of alternative host species for a pathogen.

Adding population structure can result in models that require a computational approach. For models based on systems of differential equations, the addition of various types of population structure is handled by adding additional equations. For example, age structure is commonly added by representing each age class with a separate equation. Adding equations is also an approach used to produce a model with realistic waiting time distributions for the infectious periods (Lloyd 2001). However, as the number of equations increases, our ability to derive understanding from a model analytically becomes progressively more difficult. In these cases computation becomes a rapid and efficacious approach to model analysis.

Computational modeling is also useful in making the connection between results obtained analytically and scenarios that more closely reflect real world conditions. Results derived by Meyers et al. (2003) showed that the fraction of hospital wards in which patients contract pneumonia (Mycoplasma pneumoniae) rises rapidly as the average number of wards visited by caregivers increases from 1 to 2 . This result was based on an assumption that the number of caregivers per ward and the number of wards was very large. They used a computational model to show that the result remained true for more realistic numbers of wards and caregivers.

\section{Advances in the theory of disease processes}

Extending models through computation is a powerful tool for investigating the effects of biological mechanisms on the distribution of infection. For a given epidemiological phenomenon, there may be several explanations. For example, sustained cycles in the prevalence of disease have been attributed to seasonality in host behavior (Hosseini et al. 2004), changes in rainfall or temperature (Greenwood 1987, Kim et al. 1996) or host immune response (Dowell 2001). Testing of specific hypotheses derived from these explanations can be performed quickly and inexpensively. In this section, we discuss several cases in epidemiology in which the use of computational models to explore various determinants of infectious disease has advanced epidemiological theory.

A difficulty with many early epidemiological models is that they produced unreasonably low estimates for disease prevalence during interepidemic phases (Bacon 1985, Grenfell et al. 1995). This problem can be expressed in terms of the critical community size (CSS) required to maintain an endemic prevalence of an infectious disease (Bartlett 1957). Initial model-based projections of the CCS for measles were significantly larger than estimates based on historical records (Bartlett 1957, Olsen \& Schaffer 1990). A series of models were developed to identify mechanisms that result in more reasonable estimates for the CSS. The effects of spatial structure (Grenfell 1992), seasonality (Schaffer \& Kot 1985), age structure (Bolker \& Grenfell 1993), and latency period distribution (Keeling \& Grenfell 1997) on estimates of the CCS were evaluated. Age structure and the distribution of latency and infectious periods were both shown to produce CCS estimates that are more consistent with the empirical estimate (Bolker \& Grenfell 1993, Keeling \& Grenfell 1997). 
The impact of spatial heterogeneity on disease dynamics has been shown in a number of cases to play a critical role. One form of this problem is the extent to which natural and anthropogenic sources of spatial variation contribute to the spread of disease. Smith et al. (2002) employed an elegant computational model to show that rivers, which impede the movement of raccoons, were an important factor in accounting for the spread of raccoon rabies. The same model has also been used to demonstrate the importance of long distance dispersal of infected raccoons on the spread of rabies (Smith et al. 2005a). This model was also applied in New York and Ohio, including spatial heterogeneity specific to each state, to project the spread of rabies (Russell et al. 2003, Russell et al. 2005).

The ability to handle the effects of seasonality and stochasticity efficiently with a computational model has extended the investigation of mechanisms that produce sustained oscillations in the prevalence of infectious disease. Periodic cycles in the prevalence of infectious disease are a widely observed phenomenon (Altizer et al. 2006). Some infectious diseases, such as rotavirus infection in humans, exhibit regular annual cycles in prevalence (Cook et al. 1990, Reichert et al. 2001, Rodo et al. 2002). Others, most notably measles, exhibit yearly and multiyear cycles as well as aperiodicity in the prevalence of disease (Earn et al. 2000). The effects of seasonal changes in patterns of host behavior have been extensively explored as mechanisms driving disease cycles. Computational models have been used to show that seasonal patterns of migration and aggregation in house finches can account for cycles in the prevalence of avian conjunctivitis (Hosseini et al. 2004). A deterministic SEIRtype model that includes seasonal changes in the contact rate in school-aged children can produce periodic and irregular patterns of disease outbreak that are consistent with measles (Olsen \& Schaffer 1990, Schaffer \& Kot 1985). Further modeling of measles has shown that environmental and demographic stochasticity and nonlinearity may both be important to account for the variety of periodic and aperiodic outbreaks of measles (Ellner et al. 1998). Returning to a nonstochastic model for measles, Earn et al. (2000) showed that long-term exogenous changes in the average contact rate can account for the transitions between different dynamic regimes.

Another complexity of diseases that leads to computational models is the interaction of multiple diseases or multiple strains of a single disease. For example, dengue fever has four competing strains leading to very complex dynamics. Ferguson et al. (1999) used an agestructured, multiple-strain population model to show that antibody-dependent enhancement of transmission influences the pattern of outbreaks. Additionally, co-infection with two or more different diseases can change the dynamics from what would be anticipated for a single disease in isolation. It has also been suggested that concurrent sexually transmitted infections (STI) can enhance the transmissibility of HIV. Thus, a mathematical model that includes both HIV and another STI can show the impact of controlling the spread of HIV by controlling a completely different infection (Renton et al. 1998).

Epidemiology models are often focused at the host population level, but many diseases have important dynamics at the intra-host level as the infection interacts with various tissues. For example, mathematical models have been developed and applied to give insights into the ability of Heliocobacter pylori to persist within the human stomach and cause chronic gastritis (Kirschner \& Blaser 1995, Blaser \& Kirschner 1999, Falk et al. 2000, Joseph et al. 2003, Joseph \& Kirschner 2004). Similarly, the mechanisms by which trypanosomes (Kosinski 1980), HIV (Nowak et al. 1991), and malaria (Hoshen et al. 2000) affect the host have been studied using mathematical models.

Each of the advances discussed were made possible because computational models allowed the effects of additional biological, population and environmental mechanisms to be analyzed simultaneously with great facility.

\section{Evaluation of specific intervention strategies}

Computational models can lead to changes in how we treat disease, inform public health policy and identify mechanistic linkage between disease dynamics and treatment/control strategies. 
Treatment and public health policy are truly applied problems. In some cases, formation of a meaningful policy requires that we add structure, such as space and spatial heterogeneity, to obtain an estimate of how a population will respond to a specific treatment plan. The treatment plan itself may be structured in time and space, requiring a model that can accommodate and make full use of a structured policy.

The 2001 outbreak of Foot-and-Mouth disease (FMD) in Great Britain was a significant financial blow to the country's livestock industry. The outbreak was managed by culling animals on infected premises and farms with dangerous contacts, and by restricting movement between farms. Computational modeling was used to evaluate the effectiveness of the implemented policies, as well as to evaluate the relative merits of alternative strategies. As the outbreak was unfolding, Ferguson et al. (2001b) developed a model to show that extensive culling at all farms within a geographic ring surrounding infected farms would be more effective than the existing strategy. Their model results also suggest that culling would be more effective at slowing the spread of FMD than vaccination applied to farms within a geographic ring surrounding infected farms. The results from this model influenced the subsequent management of the 2001 FMD outbreak (Ferguson et al. 2001a).

Computational models are useful for testing the effects of hypothetical intervention measures on the predicted prevalence or incidence of disease. For example, Blower et al. (2000) showed that while antiretroviral therapy will reduce the number of HIV cases and HIV-related deaths, it could also cause a change in behavior related to the perception that the disease is treatable. Blower et al. (2000) also showed that the shift in the perception could not only eliminate the net benefit of treatment but could eventually result in an increase in the number of new HIV cases. Vaccination strategies have also been evaluated using computational models. Hethcote et al. (2004) used computer simulations to compare pertussis vaccination strategies and found that changing the age for the booster shot from 18 months to adolescence would significantly reduce overall pertussis incidence.

An emerging area in computational epide- miology is the application of optimal control theory to spatially explicit models of disease spread. There are often many possible intervention measures for controlling the spread of an infectious disease. Such intervention measures might include vaccination, treatment, quarantine or behavior modification. When evaluating intervention strategies, other factors that constrain intervention measures must also be considered, such as the economic cost of the interventions. Nearly every public health effort has limited resources, and a central question is how to allocate limited resources to optimize the control of an infectious disease. That is, how can finite resources be allocated among different intervention measures, and how does the allocation change over time to produce the "best" overall control of an infectious disease? Spatial optimal control extends the optimization problem to include the effects of space and spatial heterogeneity. Asano et al. (2008) applied this technique to a model of raccoon rabies to evaluate the effects of host ecology on disease control. Raccoons are vaccinated against rabies through the periodic distribution of vaccine-laden baits. The number of baits available to raccoons changes over time as raccoons and non-target species, such as skunks, eat the baits. Ding et al. (2007) has also developed a model for the optimal spatial control of raccoon rabies to examine how vaccine dynamics change treatment policy. In this paper we discuss a new computational model that builds upon the work of Asano et al. (2008) and Ding et al. (2007) to evaluate the effects of landscape features, such a rivers, on the spatial distribution of vaccination.

\section{Novel examples}

\section{An example of simulation investigations: Evaluating alternative strategies for managing MRSA in a hospital}

As our first example, a simulation model was developed to test the projected benefits of different active surveillance (AS) strategies for methicillin-resistant Staphylococcus aureus (MRSA) control in intensive care units (ICU) (Perencevich et al.2005). Staphylococcus aureus 
is a bacterium that frequently colonizes the skin and nasal passages of humans. As the name implies, MRSA is a staph infection that is resistant to methicillin and related antibiotics. MRSA is becoming more prevalent, accounting for over $50 \%$ of all $S$. aureus infections in hospitalized patients. Individuals who are colonized with MRSA are typically asymptomatic but can transmit the disease through direct contact or to other patients on a ward via health care workers. Infections with MRSA have increased morbidity and mortality resulting in longer hospital stays and increased medical costs (Engemann et al. 2003). Isolation of known MRSA-colonized patients is a common practice aimed at reducing the probability of spread by simply reducing the number of potential contacts between colonized and uncolonized patients (Muto et al. 2003). Isolation also incurs costs, and so the question at hand is how to balance the increase costs of intervention against the potential decrease in costs from prevention of additional MRSA infections.

To begin to explore this question, we developed an individual based model for the spread of MRSA in a 10-bed ICU. The model is similar to those of Austin et al. (1999), Perencevich et al. (2004), and Raboud et al. (2005). All baseline parameters for this model are drawn from three-year averages in the University of Maryland Medical Center (Baltimore, MD, USA) medical ICU (Furuno et al. 2007). The agents in the model are the patients in the ICU and each patient has a unique id number, length of time on the ward, a true MRSA status upon admittance to the ward, a true current MRSA status, MRSA test status (not tested, pending results, false positive, true positive, false negative, true negative) and an isolation status. The ward has beds for up to 10 patients at a time and is assumed to be at capacity $98 \%$ of the time. The average length of stay, the prevalence of MRSA for incoming patients and the percent test compliance, if administered, are also known for the ward. The model runs on a 4-hour time step and is evaluated for a full year. For each time step, the probability that an uncolonized patient will become colonized, termed the "infectious pressure", of the ward is calculated based upon the patients that are present at the beginning of the time step. The infectious pressure depends on the number of colonized patients and the isolation status of those patients. If there are empty beds, there is a check to see if they are filled with new patients. Each new patient is assigned a true MRSA status based on the empirically calculated incoming MRSA prevalence. Depending upon the surveillance policy on the ward, a patient may be placed immediately into isolation upon admission. Again, depending upon the surveillance policy on the ward, a patient may be subjected to an MRSA test. Each patient is assigned a time on the ward chosen randomly from a distribution representing the observed length of stay data. For each patient with a test status of "pending", we check to see if the required time has passed for the test results and, if so, assign the test results according to the specificity and sensitivity of the test being implemented. Next, we check for any colonization events based on the infectious pressure calculated at the beginning of the time step. Finally, we check for discharge of patients based upon length of stay. This process is repeated for each time step.

This simulation model provides the structure needed to test a variety of test return times as well as basic surveillance scenarios. We evaluated many different surveillance scenarios including the following:

1. Active surveillance Type 1 (AS1): "Innocent until proven guilty." Patients are tested upon admission into the ward, but they are not moved to isolation until they have a positive test result.

2. Active surveillance Type 2 (AS2): "Guilty until proven innocent." All patients are isolated upon admission, and they are released only upon the return of a negative test result.

There are many ways to look at the results of our findings. For example, similar numbers of acquisitions of MRSA are prevented using any one of the proposed surveillance strategies. To test the robustness of these results, we completed a one-way sensitivity analysis for length of stay, sensitivity of the test results, reduction in transmission from isolation, prevalence of MRSA on admission, percentage of incoming patients tested, probability of colonization, and test return time. The two strategies had radically 
different results (Fig. 1). While the AS2 showed dramatically improved reductions in the number of new colonization events, this strategy is very cost prohibitive and thus would not be realistic for implementation in most hospitals in the USA.

Many other questions can be asked, tested and answered using this simulation model. For example, we explored the cost-effectiveness of the various surveillance strategies, calculating the cost per colonization event prevented. Some of the next steps involve expanding this simulation to include multiple wards within the same hospital and having patients transfer between wards. Additionally, other tests could be evaluated for cost effectiveness including improvements to sensitivity and specificity as well as return time.

In this investigation a computational approach takes a central role. The experiments that were performed using different strategies for isolating patients and different MRSA tests might have been unethical to carry out with real patients. In addition, had these experiments been performed in an actual hospital, considerably more time and effort would have been required to obtain similar data for analysis. The use of computers in this case allowed us to obtain insights into the efficacy of alternative treatment strategies quickly and thus allow for the rapid evaluation of a range of alternative treatment options without risk to patients.

\section{An example of computational investigations to find numerical solutions: Spatial control of raccoon rabies}

The vaccination of raccoons is used extensively to control the spread of rabies in this species and to prevent spillover into humans, domestic animals and other wildlife populations (Jackson $\&$ Wunner 2007). One aspect of the strategic use of vaccination is to incorporate the effects of landscape features, such as mountains and rivers, that inhibit or prevent the movement of raccoons (Jackson \& Wunner 2007). Rivers in particular have been shown to significantly slow the advance of rabies which can be attributed to

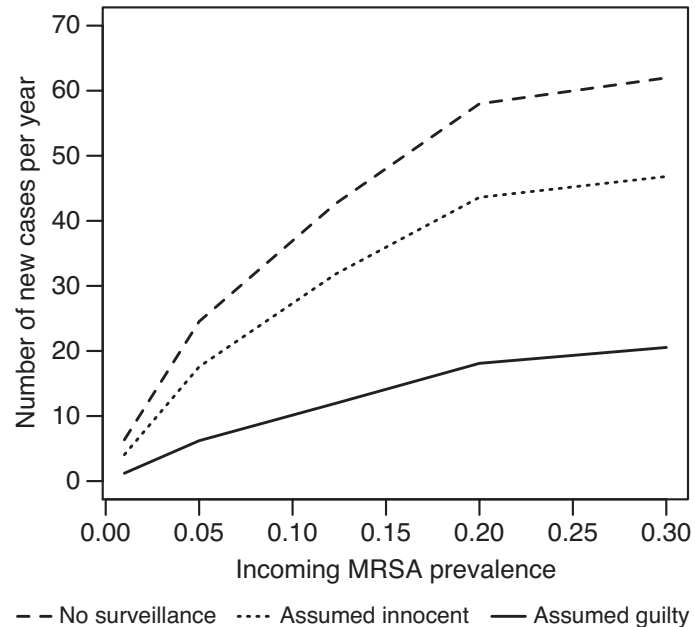

Fig. 1. Number of new colonization events for various incoming prevalence rates of MRSA under different treatment scenarios.

a decrease in the rate of short distance dispersal of raccoons across rivers (Smith et al. 2002). The spatial distribution of vaccination effort is also shaped by limited public health resources allocated to the control of infectious diseases in wildlife (Stark et al. 2006). These limitations place a premium on obtaining the best overall outcome, relative to management objectives, for the amount of investment in a vaccination policy. This section presents a model to illustrate how barriers to raccoon movement could be incorporated into an optimal rabies control policy to reduce the number of raccoon populations that must be vaccinated. The model is an extension of earlier analyses of spatial dynamics of rabies spread within the eastern US (Russell et al. 2006).

Raccoon rabies was introduced from northern Florida to the Virginia/West Virginia border in 1977 and has spread throughout the MidAtlantic States (Hanlon \& Rupprecht 1998). The westward spread of rabies has been halted by distributing vaccine-laden baits to form a cordon sanitaire, a vaccine barrier that extends southwest from Ontario, Canada into the United States, ending in Alabama (Real \& Childs 2006). The current design for the cordon sanitaire has been largely successful at preventing the spread of raccoon-variant rabies into western states. To date, the only known breach of the cordon 
sanitaire had taken place in northeast Ohio an was quickly contained (Russell et al. 2005). However, it is largely unknown how the design of the cordon relates to spatial heterogeneity. For example, it is unknown how the cordon width, the density of baits distributed within the cordon or the most effective time of year to distribute baits relates to changes in the quality of raccoon habitat or the presence of landscape features, such as rivers, that can impede raccoon movement.

Spatial optimal control is an emerging area in epidemiological modeling that combines the spatial dynamics of infectious disease and the need to make the best use of resources. Recently, several models that apply spatial optimization have been developed to address the relationship between determinants of rabies spread and the optimal strategy for vaccinating raccoons. Asano et al. (2008) developed a spatial compartment model with susceptible, exposed and infectious classes to investigate the effects of spatial heterogeneity in the disease-free population on the optimal vaccination policy. Ding et al. (2007) developed a spatial optimal control model that includes the natural attrition of vaccine baits. Once distributed, vaccine baits have a limited lifetime. Many baits are consumed by raccoons, but they are also taken by non-target species or degrade from environmental exposure. Decreasing the number of baits reduces the rate at which susceptible raccoons become vaccinated over time. Ding et al. (2007) used their model to show how optimal vaccination is influenced by natural attrition.

In this section a spatial optimal control model is used to investigate the effects of movement barriers on the optimal vaccination strategy. The optimal spatial vaccination policies are compared for a landscape with and without a river. The results from the optimal control model are also compared with related results reported by Russell et al. (2006). The comparison highlights the effects of different management objectives on vaccination policy.

The dynamics of rabies spread among raccoons is described by a system of ordinary differential equations (Russell et al. 2006). The model is based on a standard compartment based approach to disease modeling (Anderson \& May
1991) in which the population is divided into susceptible, exposed, infectious and immune classes. In addition, the model includes two age classes: juveniles and adults. The addition of age classes allows the model to include the effects of the increased death rates of juveniles relative to adults as well as differences in the movement behavior of the two classes (Stuewer 1943). The susceptible, exposed and infectious classes have interpretations typical of other SIR-type models. The immune class includes only raccoons that are vaccinated for rabies, and once vaccinated immune individuals cannot be infected. Rabies infections are always fatal to unvaccinated individuals, so there is no recovered class of individuals that have developed natural immunity (Jackson \& Wunner 2007). Space is represented by a one-dimensional array of cells, and a separate system of equations is used to represent dynamics within each spatial cell. Movement between cells involves juveniles in search of a home range. In addition, infectious individuals also move between cells as the result of behavioral changes associated with rabies (Rosatte et al. 2006). The parameter values follow those of Russell et al. (2006) and Coyne et al. (1989). The model is allowed to reach a stable diseasefree limit cycle at which point a single infected individual is added at one end of the 1-D array.

The equations for our model are:

$$
\begin{aligned}
& J_{i}^{\prime}=a(t) A_{i}-v_{i}(t) J_{i}-d J_{i}-\beta I_{i} J_{i} \\
& -M(t)\left\{\left[\phi+\mu+s e^{-\gamma A_{i}}\right] J_{i}-\phi \sum_{\ell=1}^{N} k_{i, \ell} J_{\ell}\right\} \\
& L_{i}^{\prime}=\beta I_{i} J_{i}-d L_{i}-\sigma L_{i} \\
& -M(t)\left\{\left[\phi+\mu+s e^{-\gamma A_{i}}\right] L_{i}-\phi \sum_{\ell=1}^{N} k_{i, \ell} L_{\ell}\right\} \\
& V_{i}^{\prime}=v_{i}(t) J_{i}-d V i \\
& -M(t)\left\{\left[\phi+\mu+s e^{-\gamma A_{i}}\right] V_{i}-\phi \sum_{\ell=1}^{N} k_{i, \ell} V_{\ell}\right\} \\
& S_{i}^{\prime}=-\beta I_{i} S_{i}-v_{i}(t) S_{i}-b S_{i}+s e^{-\gamma A_{i}} J_{i} M(t)(4) \\
& E_{i}^{\prime}=\beta I_{i} S_{i}-\sigma E_{i}-b E_{i}+s e^{-\gamma A_{i}} L_{i} M(t) \\
& R_{i}^{\prime}=v_{i}(t) S_{i}-b R_{i}+s e^{-\gamma A_{i}} V_{i} M(t) \\
& I_{i}^{\prime}=\sigma\left[E_{i}+L_{i}\right]-\alpha I_{i}-\psi I_{i}+\psi \sum_{\ell=1}^{N} k_{i, \ell} I_{\ell}
\end{aligned}
$$




$$
\begin{gathered}
J(0)=J_{0}, L(0)=L_{0}, V(0)=V_{0}, S(0)=S_{0}, \\
E(0)=E_{0}, R(0)=R_{0}, I(0)=I_{0}
\end{gathered}
$$

where $t$ represents time and $i$ and $\ell$ are spatial indices. The density of juveniles in cell $i$ that are susceptible, latent and vaccinated are given by $J_{i}, L_{i}$, and $V_{i}$, respectively. Adult densities in cell $i$ are represented by $S_{i}, E_{i}$, and $R_{i}$, which are the susceptible, latent and vaccinated classes, respectively. The total density of non-infectious adults is represented by $A_{i}$. Life expectancy and infectiousness are similar for both juveniles and adults (Jackson \& Wunner 2007) and their total density is represented by a single state variable, $I_{i}$. Juveniles are born at a seasonal per capita rate, $a(t)$. Raccoons are assumed to be uniformly vaccinated at a rate $v_{i}(t)$. The natural mortality rates for juveniles and adults are $d$ and $b$, respectively. Juveniles experience a higher rate of mortality as a result of differences in foraging efficiency and body size (Stuewer 1943). Susceptible raccoons become exposed to rabies through direct contact with a rabid individual. The force of infection is given by $\beta$. The seasonal movement of juveniles in the fall is represented by $M(t)$. During this time juveniles emigrate at a rate $\phi$. Juveniles immigrate into location $i$ from other locations $\ell$ at a rate $\phi k_{i \ell}$. The spatial domain of the model is closed with respect to movement so $\sum_{\ell} k_{i, \ell}=1$ for all $\ell$. We require $k_{i i}=0$, that is there is no movement from a cell to itself. Juveniles are also exposed to increased mortality associated with moving across an unfamiliar landscape (Stuewer 1943). Juveniles move in search of an area in which they can establish a home range. The suitability of a location depends on the availability of local resources and we assume that this depends on the density of adults. The term $s e^{-\gamma A_{i}}$ gives the adult density-dependent rate at which juveniles settle into a home range. The parameter $s$ is the settlement rate in the absence of adults and gamma controls the rate at which the settlement rate decreases with increasing adult density. The initial conditions for the model are given in Eq. 8.

Once exposed, a raccoon becomes infectious at a rate $\sigma$, which gives a mean latency period of $1 / \sigma$ days. Disease-induced mortality occurs at a rate $\alpha$. Since the life expectancy of rabid raccoons is short, 14 days (Coyne et al. 1989), the disease-induced mortality rate dominates other sources of mortality. For this reason, diseaseinduced mortality is the only form of loss from the infectious class. Infectious individuals emigrate out from a cell at a rate, $\psi$, and immigrate in from other cells $\ell$ at a rate $\psi k_{i \ell}$. The $k_{i \ell}$ for infectious movement are the same as those used for juveniles.

We use this model to examine the role of barriers to raccoon movement. Smith et al. (2002) has shown that there is a seven-fold decrease in the spread of rabies associated with rivers. This is interpreted as a reduction in raccoon movement across rivers. We, therefore, examine the optimum vaccination strategy produced with and without a river. The river is included in the model by designating one cell in the center of the 10 -cell array as a river. The density of raccoons in these cells is zero, and raccoons cannot enter these cells. However, occasional long-distance movement events can result in a raccoon crossing a river, for example, a raccoon could use a bridge to cross the river.

A central element of an optimization model is the formulation of an objective function. The value of this function is the quantity that is to be minimized (or maximized). It represents the management goals for the system. The objective function used here minimizes the density of infected raccoons over time and space and the total vaccination effort. The objective function used is:

$$
\min \sum_{i} \int I_{i}(t)+c v_{i}^{2}(t) d t
$$

where the value of this function is minimized over all cells $i$, and over all time, $t$. The parameter $c$ represents the relative importance of increasing vaccination relative to an increase in the density of infectious raccoons.

Since rivers are barriers to the spread of rabies we would like to test if they could be incorporated in an optimal vaccination strategy. That is, we would like to know how spatial heterogeneity affects the optimal vaccination strategy. We compare the projected optimal vaccination strategy for this objective with and without the effects of a river.

In this model, optimization is based on Pontryagin's Maximum Principle (PMP) (Kamien \& 


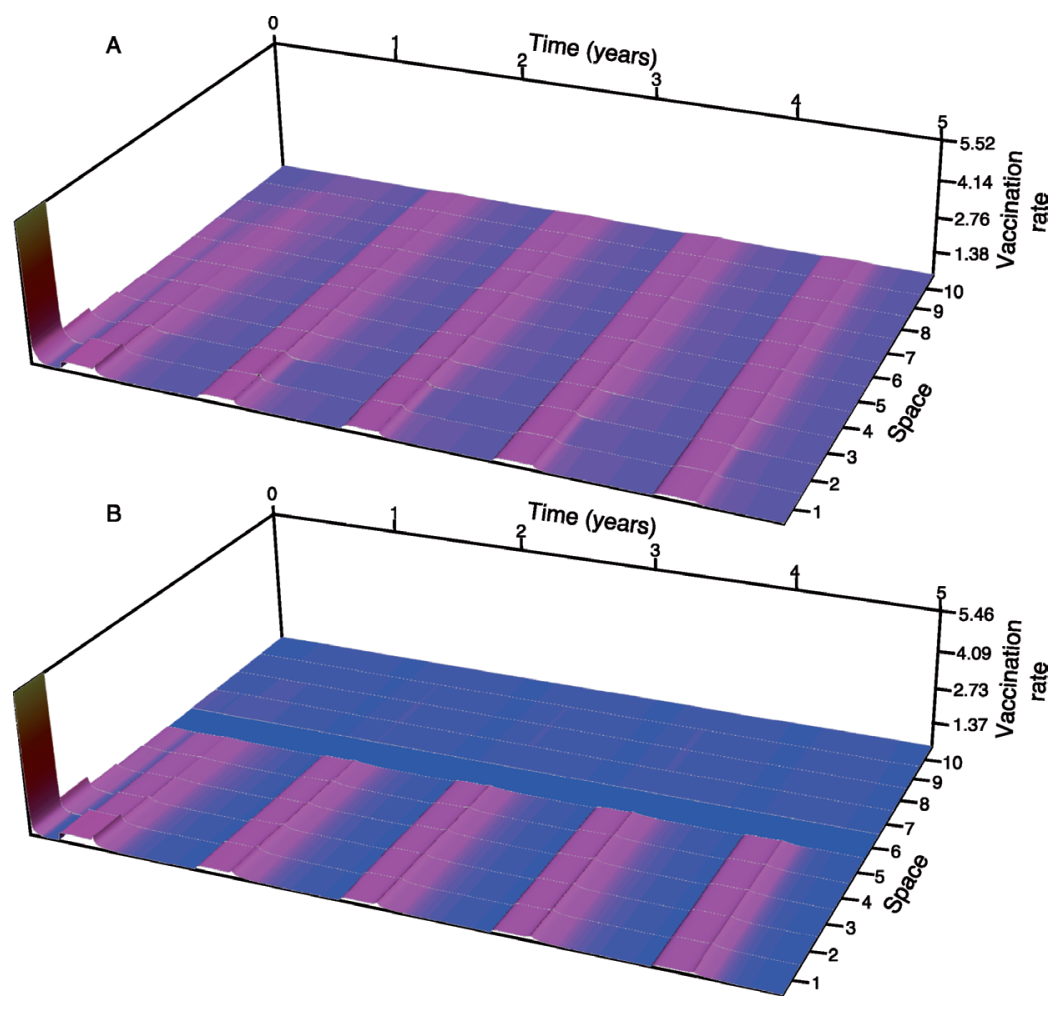

Fig. 2. Plots of the optimal vaccination strategy with and without a river. $-\mathbf{a}$ : The optimal vaccination strategy without a river. b: The optimal vaccination strategy with a river. The plots are colored according to the vaccination rates at each point, with lower vaccination rates colored blue.
Schwarz 1991). The application of this approach requires the repeated numerical integration of the model equations plus an additional system of ordinary differential equations. The extra equations, called adjoints, link the model dynamics, the objective function and the control strategy. The application of PMP requires the creation of one new adjoint equation for each of the original model equations. Since there are seven differential equations for each cell in our rabies model, seven additional adjoint equations are created for each cell. The result is a system of 14 differential equations for each spatial cell that must be solved to find the optimal vaccination strategy. Computationally, the solution involves the repeated numerical integration of the model equations forward in time and the numerical integration backward in time. The forward and backward numerical integrations are repeated until the system converges on an optimal solution. For this model, the forward and backward numerical integrations are repeated several hundred times, requiring extensive computation. Lenhart and Workman (Lenhart \& Workman 2007) give a thorough explanation of this technique, the additional mathematics required to prove uniqueness and existence of an optimal solution and the application to biological models. This approach has also been applied to compute the optimal implementation of chemotherapy to control HIV within a single individual (Kirschner et al. 1997) and to other aspects of rabies control (Asano et al. 2008).

The model results (Fig. 2) indicate that natural barriers that limit the movement of the host species result in different optimal treatment strategies. In both cases, the optimal strategy involves a vaccination rate that is initially very high followed by periodic intervals of relatively lower vaccination rates. The cyclic pattern of vaccination corresponds to the flush of susceptible juveniles created by the seasonal birth pulse. In both optimal strategies, an increase in the vaccination rate is applied over a range of cells. However, when there are no barriers to movement and the spread of the disease, the vaccination rate must be increased in all locations. In contrast, when a river is present the rate at which 
raccoons move decreases and no vaccination is required beyond the river. This has important public health implications. In particular, these results indicate that natural barriers can be incorporated into an overall intervention strategy to reduce the total vaccination rate over the entire spatial domain and thereby reduce the effort and cost of a control program.

In this example, we have presented results for an objective function that minimizes the total density of rabid raccoons plus the cost of vaccination. This is only one of a range of possible management objectives that could be used. An alternative management objective might be minimizing the month-to-month variation in the vaccination rate to achieve a more consistent plan that could be logistically easier to implement.

The importance of management objectives in shaping vaccination policy is illustrated by comparing the optimization results with the results reported by Russell et al. (2006) based on Eqs. $1-8$. The management objective for the currently presented optimization model is to find the vaccination policy that minimizes the total density of infected raccoons and the cost of vaccination. The management objective used by Russell et al. (2006) was to choose which of two vaccination policies reduced the risk of rabies crossing the river. One policy placed the vaccine barrier "in front" of the river, that is between the river and the advancing rabies wave front. The other policy placed the vaccine barrier behind the river. Of the two options, vaccinating behind the river where rabies has not yet become established resulted in the lowest risk of rabies crossing the river. This result is based on two factors. First, withholding vaccination from areas where rabies is already established results in the suppression of the overall population size and a corresponding reduction in the total number of rabid animals. Second, in the rare event that a rabid raccoon does cross the river, the rabid animal arrives in a vaccinated population. This reduces the risk of further rabies spread because the rabid animal is more likely to die before infecting a susceptible individual. In contrast, minimizing Eq. 9 requires vaccinating where rabies has become established to reduce the total density of infected raccoons. The differences in the conclusions reached from these two results highlight the importance of clearly delineating the goals of management objectives, as achieving different goals can require vastly different policies.

\section{Conclusion}

Computational modeling approaches to epidemiology have provided a number of advances in theory and practice. However, it is important to point out that in many cases, the strength of the results arising from these computational models is due in part to the incorporation of real world data. In the case of measles, the modeling program was motivated by patterns observed in an extensive record of measles cases for the US and Great Britain (Earn et al. 2000). In addition, these data sets are central to estimating model parameters and evaluating model results. The ability to map model results back to a real system significantly increases the strength of conclusions drawn from the models.

The range of models that can be considered using computers is not without limits. It is not very difficult to render a model computationally impractical. For example, a model can be made impractical by a sufficient increase in the spatial extent or resolution of a model. In many cases, the biology involved can be used to sidestep these difficulties. For example, many phenomena have a characteristic scale, and the patterns created at a large scale may not depend on processes that operate at substantially smaller scales. In others, new mathematical or numerical techniques may be developed to efficiently handle more complex problems.

Extending the range of biological mechanisms that can be concurrently modeled can also be a liability. One problem is that as the complexity of a model increases, it become progressively more difficult to understand how particular mechanisms result in the patterns produced by the model. An inability to identify the role of particular mechanisms can significantly reduce the utility of a model.

Increasing the complexity of a model entails increasing the number of free parameters in the model. Values for these parameters must be estimated. One source for estimation is to draw on results from previously published empirical 
studies. However, this approach has limitations. For example, model parameters may have to be based on distantly related species. In addition, reported estimates may not directly apply to model parameters, requiring additional assumptions to align the model with the data. Alternatively, model parameters can be estimated by fitting the model to known time series. However, as the number of parameters in a model increase, the fit to data becomes progressively better, and confidence in the estimates and model decreases (Ginzburg \& Jensen 2004). Numerous approaches exist to address these problems (Hilborn \& Mangel 1997). Careful application of these techniques can allow a more complex model to be used with a known degree of confidence.

Another challenge facing epidemiology modeling is acceptance of the modeling process and results by clinical researchers and practitioners. As clinical settings face more and more economic challenges, the use of models provides an inexpensive alternative to help focus more expensive clinical trials. Models help narrow the choice of potential hypotheses. Increased education of medical and health care workers is needed to bridge the gap and provide access to the insights that modeling can bring to questions faced by researchers.

Computational models of infectious disease epidemiology have clearly contributed to advancements in understanding the dynamics and control of many diseases. As computing power continues to improve, existing techniques will be extended in new directions and additional techniques will be created. These advances will continue to expand the questions that can be asked, provide insights into disease dynamics and help save lives by focusing limited resources on the intervention strategies predicted to make the greatest impact.

\section{Acknowledgements}

We would like to acknowledge our collaborators. David Hartley participated in the development of the MRSA model. David Smith and Colin Russell participated in the development of the rabies optimal control model. This work was supported in part by NIH grant RO1 AI047498 to LAR. Dr. Furuno was supported by NIH grant 1K12RR023250-01. Dr.
Perencevich was supported by US Veterans Affairs Health Services Research and Development Grants RCD-02-026-2 and IIR-05-123-1. Dr. Gaff was supported by Grant Number K25AI067791 from the National Institute of Allergy And Infectious Diseases. The content is solely the responsibility of the authors and does not necessarily represent the official views of the National Institute of Allergy and Infectious Diseases or the National Institutes of Health. We thank two anonymous reviewers for their valuable comments to the manuscript.

\section{References}

Altizer, S., Dobson, A., Hosseini, P., Hudson, P., Pascual, M. \& Rohani, P. 2006: Seasonality and the dynamics of infectious diseases. - Ecology Letters 9: 467-484.

Anderson, R. M. \& May, R. M. 1990: Immunization and herd-immunity. - Lancet 335: 641-645.

Anderson, R. M. \& May, R. M. 1991: Infectious disease of humans: dynamics and control. - Oxford University Press, Oxford.

Asano, E., Gross, L. J., Lenhart, S. \& Real, L. A. 2008: Optimal control of vaccine distribution in a rabies metapopulation model. - Mathematical Biosciences and Engineering 5: 219-238.

Austin, D. J., Bonten, M. J. M., Wienstein, R. A., Slaughter, S. \& Anderson, R. M. 1999: Vancomycin-resistant enterococci in intensive-care hospital settings: Transmission dynamics, persistence, and the impact of infection control programs. - Proceedings of the National Academy of Sciences USA 96: 6908-6913.

Bacaer, N. 2007: Approximation of the basic reproduction number $R_{0}$ for vector-borne diseases with a periodic vector population. - Bulletin of Mathematical Biology 69: 1067-1091.

Bacon, P. J. 1985: Population dynamics of rabies in wildlife. - Academic Press, London.

Ball, F. \& Lyne, O. 2002: Epidemics among a population of households. - In: Castillo-Chavez, C., Blower, S. M., van den Driessche, P., Kirschner, D. \& Yakubu, A.A. (eds.), Mathematical approaches for emerging and reemerging infectious diseases: models, methods and theory: 115-142. Springer-Verlag New York.

Bartlett, M. S. 1957: Measles periodicity and community size. - Journal of the Royal Statistical Society A 120: 48-70.

Biek, R., Henderson, J. C., Waller, L., Rupprecht, C. E. \& Real, L. A. 2007: A high-resolution genetic signature of demographic and spatial expansion in epizootic rabies virus. - Proceedings of the National Academy of Sciences USA 104: 7993-7988.

Blaser, M. J. \& Kirschner, D. 1999: Dynamics of Helicobacter pylori colonization in relation to the host response. - Proceedings of the National Academy of Sciences USA 96: 8359-8364.

Blower, S. M. \& Bernoulli, D. 2004: An attempt at a new analysis of the mortality caused by smallpox and of the advantages of inoculation to prevent it. - Reviews in 
Medical Virology 14: 275-288.

Blower, S. M., Gershengorn, H. B. \& Grant, R. M. 2000: A tale of two futures: HIV and antiretroviral therapy in San Francisco. - Science 287: 650-654.

Boender, G. J., Hagenaars, T. J., Bouma, A., Nodelijk, G., Elbers, A. R. W., de Jong, M. C. M. \& van Boven, M. 2007: Risk maps for the spread of highly pathogenic avian influenza in poultry. - PLoS Computational Biology 3: 704-712.

Bolker, B. 1993: Chaos and complexity in measles models: A comparative numerical study. - IMA Journal of Mathematics Applied in Medicine and Biology 10: 83-95.

Bolker, B. M. \& Grenfell, B. T. 1993: Chaos and biological complexity in measles dynamics. - Proceedings of the Royal Society of London B 251: 75-81.

Clarke, K. C., McLafferty, S. L. \& Tempalski, B. J. 1996: On epidemiology and geographic information systems: a review and discussion of future directions. - Emerging Infectious Diseases 2: 85-92.

Cook, S. M., Glass, R. I., LeBaron, C. W. \& Ho, M. S. 1990: Global seasonality of rotavirus infections. - Bulletin of the World Health Organization 68: 171-177.

Cox, N. J. \& Fukuda, K. 1998: Influenza. - Infectious Disease Clinics of North America 12: 27-38.

Coyne, M. J., Smith, G. \& McAllister, F. E. 1989: Mathematic model for the population biology of rabies in raccoons in the mid-Atlantic states. - American Journal of Veterinary Research 50: 2148-2154.

DeAngelis, D. L. \& Waterhouse, J. C. 1987: Equilibrium and non-equilibrium concepts in ecological modeling. - Ecological Monographs 57: 1-21.

DeAngelis, D. L. \& Mooij, W. 2005: Individual-based modeling of ecological and evolutionary processes. - Annual Review of Ecology, Evolution, and Systematics 36: 147168.

Ding, W., Gross, L. J., Langston, K., Lenhart, S. \& Real, L. A. 2007: Rabies in raccoons: optimal control for a discrete time model on a spatial grid. - Journal of Biological Dynamics 1: 379-393.

Dowell, S. F. 2001: Seasonal variation in host susceptibility and cycles of certain infectious diseases. - Emerging Infectious Diseases 7: 369-374.

Earn, D. J., Rohani, P., Bolker, B. M. \& Grenfell, B. T. 2000: A simple model for complex dynamical transitions in epidemics. - Science 287: 667-670.

Ellner, S., Bailey, B., Bobashev, G., Gallant, A., Grenfell, B. \& Nychka, D. 1998: Noise and nonlinearity in measles epidemics: Combining mechanistic and statistical approaches to population modeling. - The American Naturalist 151: 425-440.

Engemann, J. J., Carmeli, Y., Cosgrove, S. E., Fowler, V. G., Bronstein, M. Z., Trivette, S. L., Briggs, J. P., Sexton, D. J. \& Kaye, K. S. 2003: Adverse clinical and economic outcomes attributable to Methicillin resistance among patients with Staphylococcus aureus surgical site infection. - Clinical Infectious Diseases 36: 592-598.

Falk, P. G., Syder, A. J., Guruge, J. L., Kirschner, D., Blaser, M. J. \& Gordon, J. I. 2000: Theoretical and experimental approaches for studying factors defining the Helicobacter pylori-host relationship. - Trends in Microbiol- ogy 8: 321-329.

Ferguson, N. M., Donnelly, C. A. \& Anderson, R. M. 1999: Transmission dynamics and epidemiology of dengue: Insights from age-stratified sero-prevalence surveys. - Philosophical Transactions of the Royal Society of London Series B 354: 757-768.

Ferguson, N. M., Donnelly, C. A. \& Anderson, R. M. 2001a: Transmission intensity and impact of control policies on the foot and mouth epidemic in Great Britain. - Nature 413: $542-548$.

Ferguson, N. M., Donnelly, C. A. \& Anderson, R. M. 2001b: The foot-and-mouth epidemic in Great Britain: Pattern of spread and impact of interventions. - Science 292: $1155-1160$.

Ferrari, M. J., Grais, R. F., Bharti, N., Conlan, A. J. K., Bjørnstad, O. N., Wolfson, L. J., Guerin, P. J., Djibo, A. $\&$ Grenfell, B. T. 2008: The dynamics of measles in subSaharan Africa. - Nature 451: 679-684.

Furuno, J. P., Harris, A. D., Wright, M. O., Hartley, D. M., McGregor, J. C., Gaff, H. D., Hebden, J. N., Standiford, H. C. \& Perencevich, E. N. 2007: Value of performing active surveillance cultures on intensive care unit discharge for detection of Methicillin-resistant Staphylococcus aureus. - Infection Control and Hospital Epidemiology 28: 666-670.

Ginzburg, L. R. \& Jensen, C. X. J. 2004: Rules of thumb for judging ecological theories. - Trends in Ecology and Evolution 19: 121-126.

Greenwood, B. 1987: The epidemiology of acute bacterial meningitis in tropical Africa. - In: Williams, J. D. \& Burnie, J. (eds.), Bacterial meningitis: 61-91. Academic Press, London.

Grenfell, B. T. 1992: Chance and chaos in measles dynamics. - Journal of Royal Statistical Society B 54: 383-398.

Grenfell, B. T., Bolker, B. M. \& Kleczkowski, A. 1995: Seasonality and extinction in chaotic metapopulations. - Proceedings of the Royal Society of London B 259: 97-103.

Grimm, V. \& Railsback, S. F. 2005: Individual-based modeling and ecology. - Princeton University Press, Princeton.

Hanlon, C. A. \& Rupprecht, C. E. 1998: The reemergence of rabies. - In: Scheld, W. M., Armstrong, D. \& Hughes, J. M. (eds.), Emerging infections: 59-80. ASM Press Washington, DC.

Hethcote, H. W., Horby, P. \& McIntyre, P. 2004: Using computer simulations to compare pertussis vaccination strategies in Australia. - Vaccine 22: 2181-2191.

Hilborn, R. \& Mangel, M. 1997: The Ecological detective: Confronting models with data. - Princeton University Press, Princeton.

Hoshen, M. B., Heinrich, R., Stein, W. D. \& Ginsburg, H. 2000: Mathematical modelling of the within-host dynamics of Plasmodium falciparum. - Parasitology 121: 227-235.

Hosseini, P. R., Dhondt, A. A. \& Dobson, A. 2004: Seasonality and wildlife disease: How seasonal birth, aggregation and variation in immunity affect the dynamics of $\mathrm{Myco}$ plasma gallisepticum in house finches. - Proceeding of the Royal Society B 271: 2569-2577. 
Hyman, J. \& LaForce, T. 2003: Modeling the spread of influenza among cities. - In: Banks, H. \& Castillo-Chavez, C. (eds.), Bioterrorism: mathematical modeling application in homeland security: 211-236. Society for Industrial and Applied Mathematics, Philadelphia.

Jackson, A. C. \& Wunner, W. 2007: Rabies. - Academic Press, New York.

Joseph, I. M. \& Kirschner, D. 2004: A model for the study of Heliobacter pylori interaction with human gastric acid secretion. - Journal of Theoretical Biology 228: 55-80.

Joseph, I. M. P., Zavros, Y., Merchant, J. L. \& Kirschner, D. 2003: A model for integrative study of human gastric acid secretion. - Journal of Applied Physiology 94: 1602-1618.

Kamien, M. I. \& Schwarz, N. L. 1991: Dynamic Optimization. - Elsevier, Amsterdam.

Keeling, M. J. \& Grenfell, B. T. 1997: Disease extinction and community size: Modeling the persistence of measles. - Science 275: 65-67.

Kim, P. E., Musher, D. M., Glezen, W. P., Rodriguez-Barradas, M. C., Nahm, W. K. \& Wright, C. E. 1996: Association of invasive pneumococcal disease with season, atmospheric conditions, air pollution, and the isolation of respiratory viruses. - Clinical Infectious Diseases. 22: $100-106$.

Kim, J. J., Kuntz, K. M., Stout, N. K., Mahmud, S., Villa, L. L., Franco, E. L. \& Goldie, S. J. 2007: Multiparameter calibration of a natural history model of cervical cancer. - American Journal of Epidemiology 166: 137-150.

Kirschner, D. E. \& Blaser, M. J. 1995: The dynamics of Helicobacter pylori infection of the human stomach. - Journal of Theoretical Biology 176: 281-290.

Kirschner, D., Lenhart, S. \& Serbin, S. 1997: Optimal control of the chemotherapy of HIV. - Journal of Mathematical Biology 35: 775-792.

Kosinski, R. J. 1980: Antigenic variation in trypanosomes: a computer analysis of variant order. - Parasitology 80 : 343-357.

Lenhart, S. \& Workman, J. T. 2007: Optimal control applied to biological models. - Taylor \& Francis, Boca Raton.

Lloyd, A. L. 2001: Destabilization of epidemic models with the inclusion of realistic distributions of infectious periods. - Proceedings of the Royal Society of London B 268: 985-993.

Meyers, L. A., Newman, M. E. J., Martin, M. \& Schrag, S. 2003: Applying network theory to epidemics: control measures for Mycoplasma pneumoniae outbreaks. - Emerging Infectious Diseases 9: 204-210.

Muto, C. A., Jernigan, J. A., Ostrowsky, B. E., Richet, H. M., Jarvis, W. R., Boyce, J. M. \& Farr, B. M. 2003: SHEA guideline for preventing nosocomial transmission of multidrug-resistant strains of Staphylococcus aureus and Enterococcus. - Infection Control and Hospital Epidemiology 24: 362-386.

Nowak, M. A., Anderson, R. M., Mclean, A. R., Wolfs, T. F. W., Goudsmit, J. \& May, R. M. 1991: Antigenic diversity thresholds and the development of AIDS. - Science 254: 963-969.

O'Brien, T. F. 2002: Emergence, spread, and environmental effects of antimicrobial resistance: How use of an antimicrobial anywhere can increase resistance to any antimicrobial anywhere else. - Clinical Infectious Diseases 34: S78-S84.

Olsen, L. F. \& Schaffer, W. M. 1990: Chaos versus noisy periodicity: Alternative hypotheses for childhood epidemics. - Science 249: 499-504.

Patz, J. A., Daszak, P., Tabor, G. M., Aguirre, A. A., Pearl, M., Epstein, J., Wolfe, N. D., Kilpatrick, A. M., Foufopoulos, J., Molyneux, D. \& Bradley, D. J. 2004: Unhealthy landscapes: policy recommendations on land use change and infectious disease emergence. - Environmental Health Perspectives 112: 1092-1098.

Perencevich, E. N., Gaff, H. D., Furuno, J. P., Bradham, D. D. \& Hartley, D. M. 2005: Costs and benefits of several active surveillance (AS) strategies for Methicillin-resistant Staphylococcus aureus (MRSA) control in intensive care units (ICU). - In: 45th Annual Interscience Conference on Antimicrobial Agents and Chemotherapy (ICAAC), Washington, DC, December 16-19, 2005: 547. American Society for Microbiology.

Perencevich, E. N., Fisman, D. N., Lipsitch, M., Harris, A. D., Morris, J. G. \& Smith, D. L. 2004: Projected benefits of active surveillance for Vancomycin-resistant Enterococci in intensive care units. - Clinical Infectious Diseases 38: 1108-1115.

Raboud, J., Saskin, R., Simor, A., Loeb, M., Green, K., Low, D. E. \& McGeer, A. 2005: Modeling transmission of Methicillin-resistant Staphylococcus aureus among patients admitted to a hospital. - Infection Control and Hospital Epidemiology 26: 607-615.

Real, L. A. \& Childs, J. E. 2006: Spatial-temporal dynamics of rabies in ecological communities. - In: Collinge, S. K. \& Ray, C. (eds.), Disease ecology: community strucutre and pathogen dynamics: 168-185. Oxford University Press, Oxford.

Reichert, T. A., Sharma, A. \& Padro, S. 2001: A global pattern for influenza activity. - International Congress Series 1219: 87-94.

Renshaw, E. 1991: Modelling biological populations in space and time. - Cambridge University Press, Cambridge.

Renton, A. M., Whitaker, L. \& Riddlesdell, M. 1998: Heterosexual HIV transmission and STD prevalence: Predictions of a theoretical model. - Sexually Transmitted Infections 74: 339-344.

Rodo, X., Pascual, M., Fuchs, G. \& Faruque, A. S. 2002: ENSO and cholera: A nonstationary link related to climate change? - Proceedings of the National Academy of Sciences USA 99: 12901-12906.

Rosatte, R., Sobey, K., Donovan, D., Bruce, L., Allan, M., Silver, A., Bennett, K., Gibson, M., Simpson, H., Davies, C., Wandeler, A. \& Muldoon, F. 2006: Behaviour, movements, and demographics of rabid raccoons in Ontario, Canada: Management implications. - Journal of Wildlife Disease 42: 589-605.

Ross, R. 1911: The prevention of malaria. - Murray, London.

Russell, C. A., Real, L. A. \& Smith, D. L. 2006: Spatial control of rabies on heterogeneous landscapes. - PLoS ONE 1: e27 doi:10.1371/journal.pone.0000027. 
Russell, C. A., Smith, D. L., Childs, J. E. \& Real, L. A. 2005: Predictive spatial dynamics and strategic planning for raccoon rabies emergence in Ohio. - PLoS Biology 3: 382-388.

Russell, C. A., Smith, D. L., Waller, L. A., Childs, J. E. \& Real, A. L. 2003: A priori prediction of disease invasion dynamics in a novel environment. - Proceeding of the Royal Society of London B 271: 21-25.

Schaffer, W. M. \& Kot, M. 1985: Nearly one dimensional dynamics in an epidemic. - Journal of Theoretical Biology 112: 403-427.

Smith, D. L., Lucey, B., Waller, L. A., Childs, J. E. \& Real, L. A. 2002: Predicting the spatial dynamics of rabies epidemics on heterogeneous landscapes. - Proceedings of the National Academy of Sciences USA 99: 3668-3672.

Smith, D. L., Waller, L. A., Russell, C. A., Childs, J. E. \& Real, L. A. 2005a: Assessing the role of long-distance translocation and spatial heterogeneity in the raccoon rabies epidemic in Connecticut. - Preventive Veterinary Medicine 71: 225-240.

Smith, K. F., Dobson, A. P., McKenzie, F. E., Real, L. A.,
Smith, D. L. \& Wilson, M. L. 2005b: Ecological theory to enhance infectious disease control and public health policy. - Frontiers in Ecology and the Environment 3: 29-37.

Stark, K. D. C., Regula, G., Hernandez, J., Knopf, L., Fuchs, K., Morris, R. S. \& Davies, P. 2006: Concepts for riskbased surveillance in the field of veterinary medicine and veterinary public health: review of current approaches. - BMC Health Services Research 6: 1-8.

Stilianakis, N. I., Perelson, A. S. \& Hayden, F. G. 1998: Emergence of drug resistance during an influenza epidemic: Insights from a mathematical model. - The Journal of Infectious Diseases 177: 863-873.

Stuewer, F. W. 1943: Raccoons: Their habits and management in Michigan. - Ecological Monographs 13: 203-257.

Szklo, M. \& Nieto, F. J. 2000: Epidemiology: beyond the basics. - Aspen Publishers, Sudbury.

Weiss, R. A. \& McMichael, A. J. 2004: Social and environmental risk factors in the emergence of infectious diseases. - Nature Medicine 10: S70-S76. 